\section{The Face of Interface: Studying Interface to the Scholarly Corpus and Edition ${ }^{1}$}

\author{
Geoffrey Rockwell, Mihaela Ilovan, Daniel Sondheim, \& Jennifer Windsor \\ University of Alberta
}

Stan Ruecker

IIT Institute of Design

\section{The INKE Team}

\begin{abstract}
How can we study interface across print and digital epochs? This article proposes three ways for understanding the design of corpora across print and digital examples. We start with the idea that corpora are meant to be consulted and propose a topology of corpora, a framework of design features, and a diachronic way of studying interface. The article links to handouts ${ }^{2}$ that illustrate these ways of comparing interfaces with examples.
\end{abstract}

\section{Keywords}

Interface; Interface Research; Corpora; Print and the Web

\section{CCSP Press}

Scholarly and Research Communication

Volume 3, Issue 4, Article ID 040147, 13 pages

Journal URL: www.src-online.ca

Received March 22, 2012, Accepted March 22, 2012, Published May 30, 2013

Rockwell, Geoffrey, Ilovan, Mihaela, Sondheim, Daniel, Windsor, Jennifer, \& Ruecker, Stan. (2012). Firing on All Cylinders: Progress and Transition in INKE's Year Two. Scholarly and Research

Communication, 3(4): 040147, 13 pp.

(c) 2012 Geoffrey Rockwell, Mihaela Ilovan, Daniel Sondheim, Jennifer Windsor, \& Stan Ruecker. This Open Access article is distributed under the terms of the Creative Commons Attribution Non-Commercial License (http://creativecommons.org/licenses/by-nc-nd/2.5/ca), which permits unrestricted non-commercial use, distribution, and reproduction in any medium, provided the original work is properly cited.
VOLUME 3 / ISSUE 4 / 2012

Geoffrey Rockwell is

Director of the Kule Institute for Advanced Study,

University of Alberta. Email: geoffrey.rockwell@ualberta.ca .

Jennifer Windsor is a

graduate of Fine Arts, Visual

Communications and Design.

She is a research assistant for the interface design team of INKE.

Email: jwindsor@ualberta.ca .

Mihaela Ilovan is a graduate in Classic Archaeology, and studied Humanities Computing and Library and Information Studies at the University of Alberta. She is currently working as a graduate research assistant for the interface design team of Implementing New Knowledge Environments. Email: ilovan@ualberta.ca .

Daniel Sondheim is Lab Coordinator of the Electronic Textual Cultures Lab, University of Victora. He has a MA in Humanities Computing from the University of Alberta and is a Project Manager for the INKE project. Email: sondheim@uvic.ca .

Stan Ruecker is Associate Professor at the Institute of Design, Illinois Institute of Technology. He holds advanced degrees in English, Humanities Computing, and Design, and has expertise in the design of experimental interfaces to support online browsing tasks. Email: sruecker@id.iit.edu . 
Scholarly and Research

\section{Communication}

VOLUME 3 / ISSUE 4 / 2012

\section{Introduction}

How can we study the interface of scholarly knowledge across print and digital epochs? To ask about interface across epochs is to take a concept that makes sense in the digital world and anachronistically bring it to bear on print in a way that could confuse both. Nonetheless we need to develop ways of thinking about the relationship between design, knowledge, and audience across media, and to do that we find ourselves remediating concepts like "interface." This article takes the category of interface and adapts it to studying the design of the corpus and edition. We present three frameworks for understanding through visual comparison:

1. First, a simple framework of types of corpora that cross print and digital, which helps us organize the corpora we want to compare.

2. Second, a framework of types of interface features used in the design of corpora and editions that are abstracted from print and digital examples.

3. Third, a framework for diachronic study of interface.

This article is, in effect, a survey of the analytical frameworks we have developed over the last two years in the Interface Design group of Implementing New Knowledge Environments (INKE) at the University of Alberta. ${ }^{3}$ These frameworks are not arguments about the history of interface, but structures for comparing in order to develop hypotheses. In effect, we have to develop the categories and practices of comparison in order to be able to draw inferences. To that end,

1. We gather both analogue and digital examples with the help of the INKE Textual Studies group. Gathering samples goes hand in hand with defining what it is we are studying.

2. We try to see if there are logical groupings in the collection of examples across media rather than groupings by media. The reason for this is to try to understand the continuum of interface. These groupings could come from the literature, or from the intended audience.

3. We then look closely at the sample interfaces, asking if there are patterns of similar or related features. In particular, we are interested in features that cross from print to digital, but also in those that seem specific to one or the other medium. Hypotheses are developed and discussed by the team.

4. Finally, we pick particular works that have gone through a number of editions to look diachronically at how the interface changes incrementally and ask about the decisions that led to those changes.

5. Examples go into a database with a rich prospect interface ${ }^{4}$ so that we can incrementally survey examples we have gathered and develop further hypotheses and frameworks. 


\section{Topology of corpora and their audiences}

In order to ask about the interface of the corpus, we found that we needed to develop a working topology of what it was we would study. We wanted a sense of what a corpus is and what the major types of corpora are, on the assumption that interface might vary across types. At first glance, any collection of items could be interesting to consider as a corpus for the purpose of exploring interface, including how operating systems present folders of icons of files. To make matters more interesting, computing and the database are changing our notion of what a collection is. As Lev Manovich (2001) points out in The Language of New Media, the random access database is to new media as narrative is to film. In "The Database as a Symbolic Form" he writes,

Many new media objects do not tell stories; they don't have beginning or end; in fact, they don't have any development, thematically, formally or otherwise which would organize their elements into a sequence. Instead, they are collections of individual items, where every item has the same significance as any other. (Manovich, 2001, para 1)

The database that structures items so that they can be retrieved and organized in different sequences called reports could be seen as the digital extension of the collection or corpus, but this leads to problems if we want to use interface beyond new media.

First, databases would seem to be the logic behind new media and this logic creates the conditions for interface. There is a separation of content from visible form: this logic separates the data and technical organization of the database from what the users sees - the interface. The database is a structure of information that has abstract form that can only be accessed through an input and output interface. The corpus as database therefore has a particular relationship to interface that it doesn't necessarily have in analogue corpora where the physical form and content are one.

Second, the database has no particular arrangement, as it is logically a random set of structured items. A print corpus, even if it is meant to be consulted randomly, will have an organization imposed by its material form. There will be a first record and a second. That organization would seem to be part of its interface. A database is the logical extension of consultation where the physical arrangement (on a hard-drive) is hidden in favour of a logical or structural arrangement. The database is logically independent of the interface in order to facilitate multiple interfaces. Where a print corpus will have one privileged physical interface - the alphabetical arrangement of entries, for example - the database hides the physical organization on the hard drive in order to present the possibility of any interface. While the print corpus may make use of indexes, tables of contents, and other aides to overcome the physical arrangement and provide alternative interfaces, the database forces the programmer to build an interface of some sort, even if it is just to get data into the database.

What remains the same, however, is the use and audience of print and digital corpora. The database can be used for consultation in much the same way as the corpus. Ann Blair in Too Much To Know proposes the idea of "consultation reading" as the way readers used corpora and other types of scholarly documents like the scholarly edition.
VOLUME 3 / ISSUE 4 / 2012 
Scholarly and Research

\section{Communication}

VOLUME 3 / ISSUE 4 / 2012
Consultation reading existed amongst the learned in earlier centuries, and in an unbroken line of transmission at least as far back as the thirteenth century, so that the most distinctively new kind of reading in the eighteenth century was not consultation reading but rather engrossment in the novels that were a new and successful genre. (Blair, 2010, p. 59)

We therefore used consultation as a guide to the question "What is a corpus?" and looked to who would use a corpus to categorize corpora. Reviewing the variety of corpora used by scholars, we noticed three types that appear in both print and on the web, which seemed to have similar audiences and uses, and a fourth catch-all category. This gave us a framework or topology for looking at the interfaces of corpora. We then created a large-format handout ${ }^{2}$ with a grid of the three types across as columns and Print and Web for the rows. This grid is a visual representation of this first framework and we use it to compare across types and media. Different examples can be put in the cells for visual comparison. Following is an explanation of the types. You can see the PDF of this framework at http://inke.ca/wp-content/uploads/2012/11/topology.pdf .

\section{LINGUISTIC CORPORA}

Linguistic corpora, or collections of language use, are important to the field of linguistics, especially (it won't surprise you) corpus linguistics. While these corpora are mostly digital, because they are so much more useful to linguists in digital form, there are examples of print linguistic corpora.

Linguistic corpora dramatically show how computation has changed the interface of the corpus. Linguistic corpora, like the Corpus of Contemporary American English, are designed not for reading but for statistical searches. ${ }^{5}$ Unlike print corpora or even digital corpora in other fields, digital linguistic corpora hide the original text and privilege charting through searching, comparison, and statistical tools.

\section{Artefactual Corpora}

Artefactual corpora document collections of objects of scholarly interest like coins, pottery, or buildings. One thing that stands out immediately about such corpora is that they aren't surrogates for the object collections the way you could say a literary corpus can act as a surrogate for the original. Instead, they collect information about a distant object that allows the scholar to study it remotely and to compare it with other such objects in ways you couldn't do physically. While it would be interesting to discuss the ontological status of the things collected in a corpus (what is a literary thing in itself?) our point is about the interface. In both print and digital corpora of objects, a major interface element is the attempts to show the object to a scholar who can't be there. A corpus of inscriptions might include a photograph and a cartoon drawing to help the scholar who can't physically trace the writing that is hard to see in the photograph. Similarly, we see digital collections using multimedia to allow users to zoom in on a detail of a coin or zoom out to see a rich prospect view of the whole collection the way you might see a tray of coins in a museum. In other digital collections we see video clips of objects like art books being opened or "virtual" dresses where you can turn or zoom in on a $3 \mathrm{D}$ model of an historic dress. 
Artefactual corpora show how many scholarly digital corpora have remediated preexisting collections of information. Research coin collections have been around for a while and you can see how they already have the structure of a database where there are consistent fields for each record. The curator of the Bruce Brace Coin collection at McMaster (Bruce Brace himself) developed a card filing system, which structured the information gathered about each coin. When the team built the database for the web version, they used the same structure (with a few more fields) and transcribed the data. ${ }^{6}$ The problem with the cards, of course, was that you couldn't search on any field and had to know all the abbreviations used by Brace to use the cards. The cards were a curator's tool, whereas the online database is a public research tool.

\section{LITERARY CORPORA}

Literary corpora are collections of literary works, often complete sets of a particular author or a collection of works of a genre like poetry from a particular language and period. Unlike linguistic corpora, they are meant to be read sequentially in addition to being consulted. The texts are not hidden behind a search engine, instead, such digital corpora will often use tables of contents and indexes as the primary ways into the content. More than any other type of corpus, digital literary corpora resemble their print cousins, often remediating the form instead of reinventing it. This is perhaps because they collect fewer items and because the items collected are important in themselves in a way that a linguistic fragment or image of a pot shard is not. The user first finds the item using one set of interface features that we call organization and navigation aides, and then uses a different set of features within a record. There is thus in literary corpora, but also in artefactual corpora, in both print and digital, a separation between the finding interface (what you use to find an item in the collection) and the reading interface (which you use to carefully study the item).

Of particular interest to us are the variety of reading interfaces in print corpora and how they have been adapted to the Web. In print, designers have to deal with limited space and have to balance the desire to have a clear reading experience against the need to provide scholarly annotations close to the representation; in digital, the designer can provide multiple views of the same content. One can, for example, open parallel columns so that page images can be seen and synchronized with annotated text. The luxury of being able to solve design problems by just multiplying views and providing more controls has been irresistible to digital designers of scholarly corpora, but these views have their predecessors in print. We suspect that in the interests of easeof-use we will begin to see standard arrangements of materials so that scholars can concentrate on the evidence and ignore the interface doodads.

A rough fourth type of corpus not used in our framework or illustrated in our handouts ${ }^{2}$ is, for lack of a better term, the normative corpus. Examples would be collections of laws, collections of religious materials, and other collections used to suggest how people should act. We did not include this in our topology because it is not a coherent type, but a catch-all for corpora that have a common normative aim.

\section{Framework of interface features}

The second framework we have developed is for the types of features used in the design of corpora and scholarly edition interfaces. This framework is one way of categorizing
VOLUME 3 / ISSUE 4 / 2012 
Scholarly and Research Communication

VOLUME 3 / ISSUE $4 / 2012$ the affordances that the designer can deploy. We have tried to develop a framework that allows for comparison across print and the Web - this framework was drawn from both - but some of the types of features betray their origins. For example, we had to deal with the problem of physical organization being part of the print interface in a way that it could really be on the Web. Perhaps with further thought we will be able to collapse classes of features into more abstract classes that are independent of medium, but for the moment this framework serves to help think through what is going on across page and screen.

These broad interface categories include the (physical) organization of the corpus, the navigation aides, paging devices, record layout, and textual features. Again, we developed a handout ${ }^{2}$ to illustrate how one can arrange features using this framework. Again, this framework is not a conclusion about interface so much as a framework for comparing with which to develop hypotheses. You can see the PDF of this framework at http://inke.ca/wp-content/uploads/2012/11/framework.pdf .

What unifies these features is that they represent different ways for the designer to help the user "consult" a work. Consultation as discussed by Ann Blair (2010) involves a number of activities including

- finding a particular item

- checking a fact or a hunch

- searching for something in response to a query

- browsing around an issue from a starting point

- browsing to survey a topic

- comparing items that are similar in some way

- serendipitously wandering from topic to topic

- cramming for an exam by consulting all the items you might be examined on

We should note that consulting is not the same as searching; consulting might involve searching through a collection, but in consultation, the searching is followed by skimming results and then close reading of relevant items. Given the size of collections and the type of content, users rarely read the complete collection from start to finish (or in any order). And given that most readers don't know the contents well, a lot of design goes into helping users locate items. We therefore find both print and digital corpora rich in design ideas for such finding, checking, browsing, and comparing. Here are the major groups of features we found that seem to cross media, even if some originate in print or the web. The first page of the handout ${ }^{2}$ details features that are for reading records, the second page details features for finding records.

\section{RECORD LAYOUT}

Record layout is the design of how an item is displayed. We found that designers tend to keep the layout consistent to aide consultation. If you are looking for a particular field a consistent layout helps your eye jump to the desired area. In print the record doesn't always correspond to a page. A corpus of coins might have a couple of entries per page so there also have to be ways to distinguish the end of one record and the start of another. 
Print layouts tend to be more complex because the designer doesn't have to account for pages being resized and there is more usable resolution on a page than on most screens. Digital designers, on the other hand, can use different views to show more or less of a record.

\section{Textual Features}

Textual features such as colour, drop caps, and superscripted footnote numbers are used in both print and digital to convey information not only about content, but also about navigation in the corpus. Think of how headwords are bolded and how the size of the text often indicates its potential importance to the user.

In digital corpora we find some unique uses of textual features like the yellow dynamic highlighting of search words found on a screen. We see combinations of features from background colour, text colour, text font, and emphasis used to differentiate the words used for navigation from those that are content. Look at the Web example in the handout ${ }^{2}$ from the online version of the Index Thomisticus and note all the different text styles used for different elements of information.? Such variety would be expensive to print, which is why we often find print designers using layout more.

\section{Organization}

Organization of the whole corpus, whether physical or virtual, is an important starting point for anyone consulting a collection. Print corpora may be broken into volumes or be bound in one large volume. That a concordance is arranged in alphabetical order is important to know if you are going to use it. The alphabetical arrangement of the precursors to the concordance was an important design idea that changed what could be done with them (Rouse \& Rouse, 1974.) You didn't need to know how the subjects were organized to find something, and you could browse around to get ideas. Given the importance of the arrangement, it is common to have physical affordances like tabs that help the user use that arrangement.

In digital works the separation of database from interface means that the designer can invent a virtual arrangement based on alternative physical analogues. This was more popular in the early days of the Web when many scholarly works took some feature of the phenomenon as an interface idea. For example, the Valley of the Shadow site uses the floor plan of Jefferson's central building at the University of Virginia. ${ }^{8}$ The idea is to create a familiar interface myth like the desktop of the Macintosh that helps users navigate as if this is just an extension of what you know. Now designers can count on more educated users who expect a certain consistency with similar sites, rather than site specific navigation aides. The cost of maintaining the interface myth is too high and frequent users get tired of the design ideas generated by textual scholars.

\section{NAVigation AIDES \\ "Navigation aides" is our term for the tables of contents and indexes that document and supplement the physical arrangement. In print you can have only one organization of the materials, but you can supplement the arrangement with multiple indexes, in effect providing alternative interfaces to the same materials. This is especially important to artefactual corpora, which don't easily sort in some fashion the user would know. Digital interface designers have drawn heavily on the innovative types of}

VOLUME 3 / ISSUE 4 / 2012 
Scholarly and Research Communication

VOLUME 3 / ISSUE 4 / 2012 indexes developed by print designers. The difference is that the digital designers can automate the generation and linking of these indexes.

In digital works, given the separation of interface from database, there is nothing but designed navigation aides, as the physical arrangement of the information on a hard drive of a server is useless to the user. The example on the handout ${ }^{2}$ shows a table of indexes for a collection of images of Trajan's Column. ${ }^{9}$ Such indexes also provide a useful sense of the scope of the whole corpus to the user and what its purpose was. You can tell, by the choice to tag the images with that information, that many of the images of Trajan's Column were taken to document tool marks. As we will see in the next section, interfaces reflect not only what a designer anticipates about the user, but also the choices by the authors. This gets us back to what makes a corpus. A corpus is not any collection of items, but one that has a logic - a collection gathered for some research purpose. That purpose informs the choice of what to collect, how to document it, and how to create a useful interface.

\section{Paging Devices}

Paging devices are used to provide context as to where a user is in a collection in a way that connects to the organization and navigation paradigms. A running head in a print corpus usually provides a lot more information than just the page number. It usually provides information about where you are in the corpus along with key information about the items on the page. This can connect to the organizing navigation aides. You use the table of contents to get to the neighbourhood of what you want and then the running head to find the right page.

Digital works will likewise show some contextual information, sometimes in the title bar of the window, but also on the page. In digital works, what is different is that there is usually more space dedicated to a common navigation bar. In print you might have just the title of the corpus in the running head, but in the digital format you usually have a common navigation tool bar, which can be used to get back to the home page, get to indexes, or allows you to search. There is also significant space dedicated to branding of the page as belonging to $\mathrm{X}$ project. This is because on the Web, a link can take you from one collection to another; moving seamlessly to a different work.

\section{Framework for diachronic study}

Lastly we introduce a framework for understanding change in interface over time. The features framework is for synchronic comparison, but how does one compare the successive design changes of a long-running project like the Perseus Project? ${ }^{10}$ What makes it even more difficult is that change in the interface of a work is often due to designers adapting to changes in the mediating technology, changes in the presumed users of a work, changes in the mission of the scholarly work, or changes in the culture of the media. We need a way to study a history of interface that lets us make inferences about change.

To that end, we have been studying print and digital corpora that went through many editions just as the Perseus Digital Library did, which first came out on CD-ROM and then on the Web. For such diachronic study we have developed both large-format representations of key interfaces over time (see a handout ${ }^{2}$ related to the study at http:// inke.ca/wp-content/uploads/2012/11/diachronic.pdf), but found it more useful to 
animate the change over time. Instead of a handout we recommend an animation of the evolution of the homepage interfaces we have been able to recover. This can be scrubbed forward and backward to visually follow and check change. See the YouTube video "Perseus Digital Library Diachronic View (University of Alberta)" at http://www. youtube.com/watch?v=WE4zg8TCMiQ .

\section{Figure 1: Portion of the Perseus Homepage 1997}

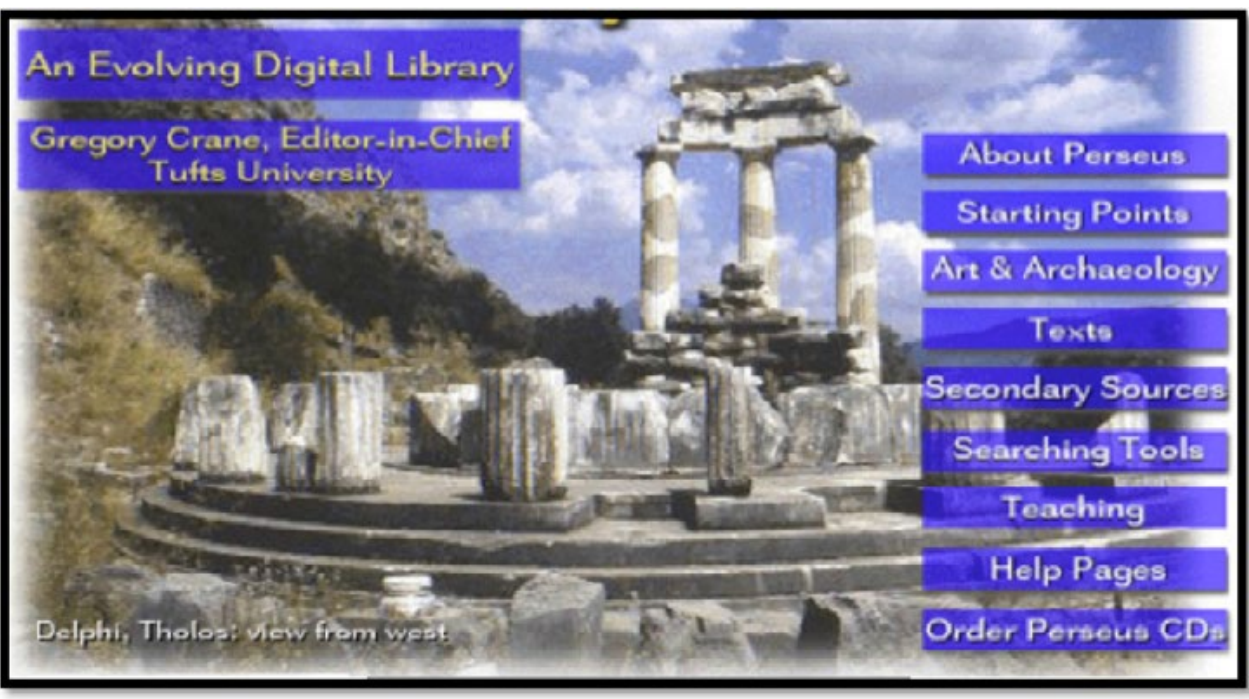

Figure 2: Reconstituted timeline of the Perseus project

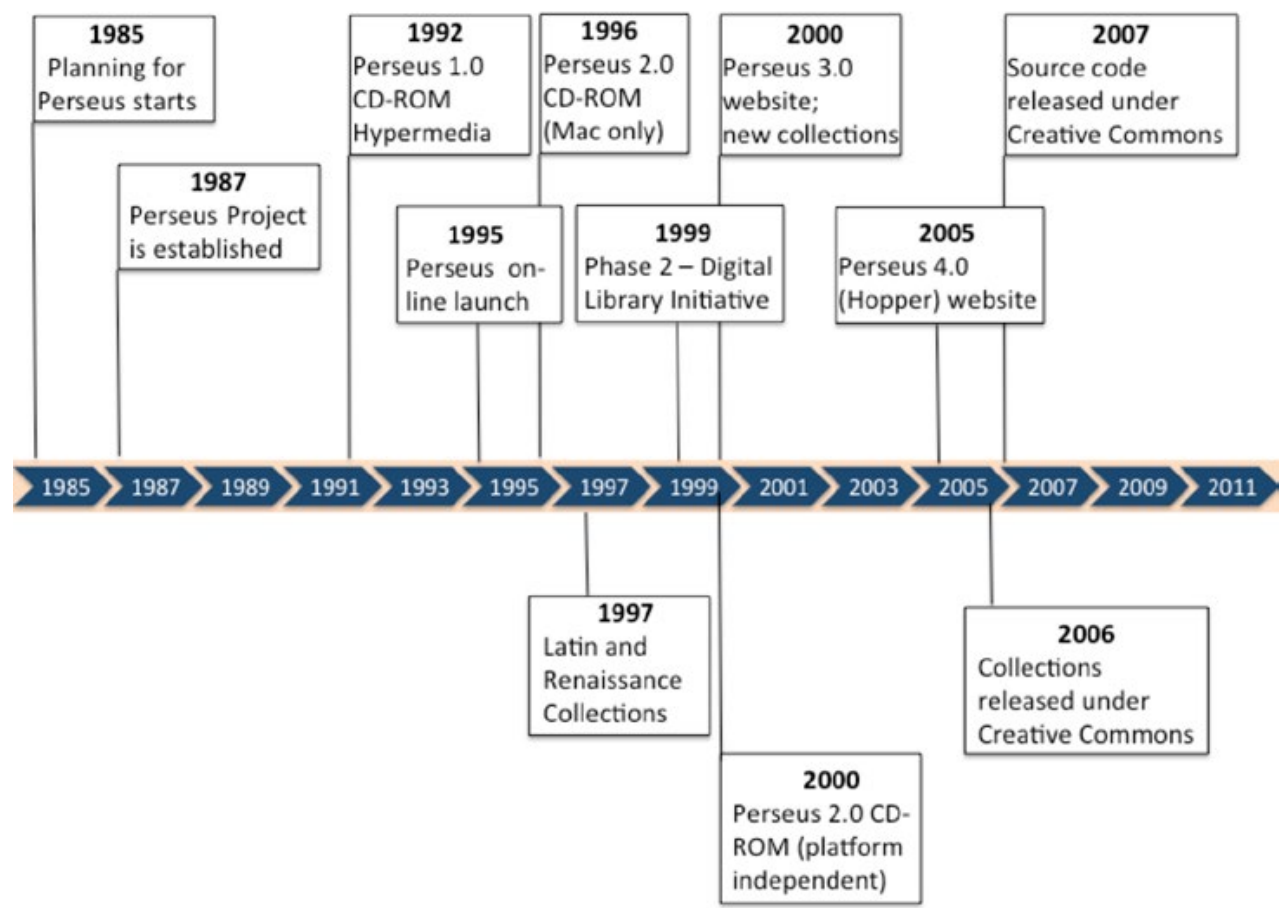

Rockwell, Geoffrey, Ilovan, Mihaela, Sondheim, Daniel, Windsor, Jennifer, \& Ruecker, Stan. (2012). Firing on All Cylinders: Progress and Transition in INKE's Year Two. Scholarly and Research Communication, 3(4): 040147, $13 \mathrm{pp}$.

\section{Scholarly and Research}

\section{Communication}

VOLUME 3 / ISSUE 4 / 2012 
Scholarly and Research

\section{Communication}

VOLUME 3 / ISSUE 4 / 2012
How does one document interface change? Given how little is written about interface and how projects don't necessarily document their changing interfaces, we have used the Internet Archive Wayback Machine to gather images of the homepage of Perseus over time. ${ }^{11}$ Figure 1 shows an early interface while Figure 2 shows our reconstituted timeline of the project based on documentation in sites and what we could gather.

Once we gathered and animated the evolution of the Perseus interfaces, we found the question was about how to understand the changes. To that end we developed a simple framework of things to look for and questions to ask about change. Asking questions, we developed inferences about the changes we saw that in some cases we are able to check with members of project.

\section{Mediating TeChNOLOgY}

Interfaces can change dramatically when the mediating technology changes, as in the case of the change in Perseus from HyperCard on a CD-ROM to the Web. HyperCard enforced a "card" size and didn't support colour, while Web pages are designed to scroll, the window can be resized, and they can have colour. One of the first questions we ask when reading change in an interface is whether there has been a change in the technology.

A more common change in technology in the evolution of Web interfaces is the availability of larger and larger screens and greater colour-depth. This is harder to track as designers are making decisions about what sort of display they think their audience has. Even when large screens are available, a designer might aim for a school market where there are still mostly smaller screens and 8-bit colour. Nonetheless, over time we can see designers developing interfaces optimized for wider screens. For example, we see the addition of columns of information that assume there is enough space for at least two readable columns.

\section{ChANGES IN INTENDED AUDIENCE}

In some cases, a change in interface can reflect a change in the intended audience of the work. One needs to be careful about such inferences as they are really a doubled inference in the sense that one is inferring a change in the intentions of a designer who is inferring intentions in a class of users. Nonetheless, one can see changes that are documented by the project itself. For example, one can see in the project that Perseus starts out as a corpus designed for teaching classics and, at a certain point, changes to become a "library" that includes collections of texts that are not classical. Around the year 2000, perhaps because they received grants to digitize materials about other periods and regions, Perseus changes its name from the Perseus Project to the Perseus Digital Library.

\section{CHANGES IN THE MISSION OF THE SCHOLARLY WORK}

Similar to changes in intended audience, we also see evidence of changes in mission. One could say that a change in intended audience is a change in mission, but here we want to distinguish between changes in who a corpus is intended for from changes in what sort of consultation use is supported, even if by the same audience. These changes are often seen in the major menu headings of a site, changes that reflect what the designer thinks are the major uses of the work. One can also find explicit text in About or Mission pages that describe what the purpose of the corpus is. 
Changes in mission also appear in the interface - the designers "show" new ideas to us. In 2000, Perseus added geospatial features and introduced those with a time/space visualization that appears in the centre of the interface (see Figure 3).

Figure 3: A graph of the places and dates mentioned in Perseus

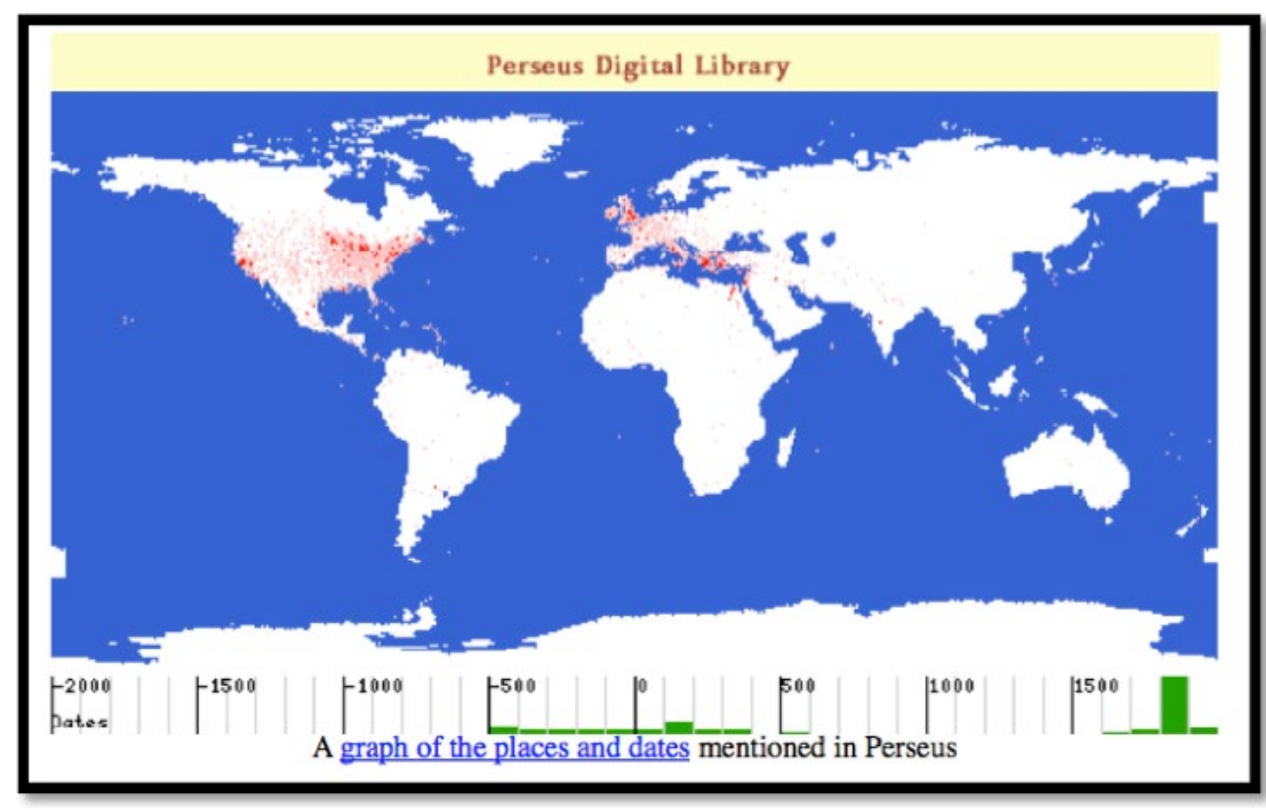

\section{Changes in the Culture of the media}

Lastly, we have changes in the design culture. Websites will have their interfaces refreshed not because of any change in mission or technology, but simply to make them look fresh and current. There are fashions to interface such that a site, no matter how useful, can look out of date. Projects that have access to designers will update their interface to visually indicate currency. The design of an interface can tell users subtle things about whether the project is ongoing or whether it was frozen at a certain point.

The interface to a scholarly work mediates between the authors/designers and audience of a work. Changes in the interface can be read as a log of changes between whoever is driving the project and the audience. She may be adapting to changes in the technology she thinks the user has, or changes in who she thinks the user should be. She may be changing the interface to respond to changes in the content and mission of the project or she may be simply keeping the interface attractive and fresh. Whatever the reasons, the log of changes between interfaces is a fascinating text hinting at the ongoing negotiation of information. Here we have talked about following a digital interface over time, but it is possible to track print interfaces from edition to edition too.

\section{Conclusion}

To conclude, we feel we have just scratched the surface of the interface of the scholarly work. The corpus and scholarly edition are types of documents that have an overabundance of information that is consulted, but not necessarily read intensely cover to cover, the way we read a novel. Whether in print or on the Web, the interface is important in the face of this excess, as it is how the designer helps the user understand the scope of what can be found and consulted. 
Scholarly and Research

\section{Communication}

VOLUME 3 / ISSUE 4 / 2012
Interface is important not only because it communicates about the work and makes a collection easy to use, but it can also enable or disable novel uses of a work and new ways of thinking through works.

The use of alphabetical order was a tacit recognition of the fact that each user of a work will bring to it his own preconceived rational order, which may differ from those of other users and from that of the writer himself. Applied to distinction collections, this notion meant recognition that, while one might teach in the order of the text of the Bible, one did not preach thus. Applied, for example, to the Bible itself, this notion produced the verbal concordance. Alphabetization was not simply a handy new device; it was also the manifestation of a different way of thinking (Rouse \& Rouse, 1982, p. 212)

Just as the alphabetical arrangement of a concordance enabled a new style of preparing to preach, likewise the processing available on a computer has enabled corpus linguistics to use corpora differently for statistical research. Because interfaces reflect and enable ways of thinking through texts, we need to pay more attention to analyzing them. This article has proposed frameworks for that analysis, not conclusions; we have been experimenting with ways of presenting research about interface both within our team and to others. The handouts ${ }^{2}$ that accompany this article and the animation of the evolution of the Perseus interface on YouTube are experiments in how to study interface. Dissatisfied with the space of the article, we wanted to play with the interface to the study of interface.

\section{Notes}

1. Article for INKE BOF Research Foundations for Understanding Books and Reading in the Digital Age. Portions of the article were presented as a short paper at the Digital Humanities 2011 conference at Stanford University (https://dh2011 .stanford.edu) and Society for Digital Humanities (SDH/SEMI) 2011 conference at the Humanities and Social Sciences Federation of Canada (HSSFC) Congress at the University of New Brunswick, Fredericton (SDH-SEMI is now the Canadian Society for Digital Humanities, http://csdh-schn.org).

2. The links to the handouts are as follows: http://inke.ca/wp-content/uploads/2012/11/framework.pdf http://inke.ca/wp-content/uploads/2012/11/topology.pdf http://inke.ca/wp-content/uploads/2012/11/diachronic.pdf

3. For more on INKE and the INKE team see the website INKE: Implementing New Knowledge Environments (http://inke.ca).

4. A rich prospect is a view that shows some meaningful representation of every item surveyed. See Ruecker, Radzikowska, and Sinclair (2011).

5. See The Corpus of Contemporary American English (COCA) website (http:// corpus.byu.edu/coca). 
6. To see and read about the Bruce Brace Coin Collection see http://tapor1.mcmaster .ca/ coins.

7. For the Index, see Bernot and Alarcón (2005).

8. For the Valley project see the website The Valley of the Shadow: Two Communities in the American Civil War (http://valley.lib.virginia.edu).

9. See the Trajan's Column website (http://cheiron.mcmaster.ca/ trajan).

10. See the current website for the Perseus Digital Library (http://www.perseus.tufts.edu).

11. See the Wayback Machine website (http://www.archive.org).

\section{Websites}

Bruce Brace Coin Collection at McMaster University. URL: http://tapor1.mcmaster.ca/ coins

Canadian Society for Digital Humanities / Société canadienne des humanités numériques. URL: http://csdh-schn.org/

Digital Humanities 2011: June 19-22. URL: https://dh2011.stanford.edu

INKE: Implementing New Knowledge Environments. URL: http://inke.ca

Perseus Digital Library. URL: http://www.perseus.tufts.edu

Perseus Digital Library Diachronic View (University of Alberta). URL:

http://www.youtube.com/watch?v=WE4zg8TCMiQ

The Corpus of Contemporary American English (COCA). URL: http://corpus.byu.edu/coca

The Valley of the Shadow: Two Communities in the American Civil War. URL:

http://valley.lib.virginia.edu

Trajan's Column. URL: http://cheiron.mcmaster.ca/ trajan

Wayback Machine. URL: http://www.archive.org

\section{References}

Bernot, Eduardo, \& Alarcón, Enrique. (2005). Index Thomisticus.

URL: http://www.corpusthomisticum.org/it/index.age [June 18, 2012].

Blair, Ann M. (2010). Too much to know: Managing scholarly information before the Modern Age.

New Haven, CT: Yale University Press.

Manovich, Lev. (2001). The language of new media. Cambridge, MT: MIT Press.

Manovich, Lev. (2001). Database as a symbolic form. URL: http://transcriptions.english.ucsb.edu /archive/courses/warner/english197/Schedule_files/Manovich/Database_as_symbolic_form.htm [June 17, 2012].

Rouse, Richard H., \& Rouse, Mary A. (1974). Biblical distinctiones in the thirteenth century. Archives D’Histoire Doctrinal et Littéraire du Moyen Age, 41, 27-37.

Rouse, Richard H., \& Rouse, Mary A. (1982). Statim invenire: Schools, preachers, and new attitudes to the page. In Benson, Robert Louis, \& Constable, Giles (Eds.) Renaissance and Renewal in the Twelfth Century (pp. 201-225). Toronto, ON: Harvard University Press.

Ruecker, Stan, Radzikowska, Milena, \& Sinclair, Stéfan. (2011). Visual interface design for digital cultural heritage: A guide to rich-prospect browsing. Farnham, Surrey, UK: Ashgate Publishing.
Scholarly and Research

Communication

VOLUME 3 / ISSUE 4 / 2012 